\title{
Solution of periodic heating problems by the transfer matrix method
}

\author{
Shiqing Fan, J.R. Barber * \\ Department of Mechanical Engineering, 2250 GG Brown Laboratory, University of Michigan, Ann Arbor, MI 48109-2125, USA
}

Received 18 February 2001; received in revised form 30 June 2001

\begin{abstract}
For discretized linear periodic heating problems, the nodal temperatures at the end of a heating cycle must be a linear function of those at the beginning of the same cycle and of any reservoirs with which the system exchanges heat. If the matrix of coefficients defining this system of linear equations is determined, it can be applied sequentially to advance through the transient response to the system without the need for direct numerical simulation of every cycle. It also permits a direct solution for the nodal temperatures at an appropriate time during the steady periodic state. Methods of determining the required matrices are discussed and the method is applied to a simple example. (C) 2002 Elsevier Science Ltd. All rights reserved.
\end{abstract}

Keywords: Periodic heating; Transfer matrix method; Finite element method

\section{Introduction}

Many situations exist in which systems are subjected to periodic heating and cooling. For example, during the combustion cycle, the valves of an internal combustion engine experience direct heating from the combusting gases, cooling from intake air and periods of thermal contact with the valve seat [1]. Other examples include heat conduction in sliding solids [2], regenerative heat exchangers [3], solar heating systems [4] and heat conduction between the workpiece and the die in repetitive forming and rolling processes.

Transient behavior of such systems will consist of a gradually evolving periodic perturbation superposed on a long term trend which generally tends to a steady state. In some cases interest will focus mainly on the steady periodic solution, but in some applications this will not be reached during the normal operating cycle and hence the transient problem must be solved.

\footnotetext{
${ }^{*}$ Corresponding author. Tel.: +1-734-936-0406; fax: +1-734647-3170.

E-mail address: jbarber@umich.edu (J.R. Barber).
}

Numerical solution is required in all except the simplest (e.g., one-dimensional) examples and the obvious way to proceed is to use numerical simulation [5] - i.e., to step in time solving at each step for the instantaneous temperature field (nodal temperatures). However, this is very time consuming, particularly if many cycles of heating are to be analyzed.

An alternative method can be developed when the governing equations and boundary conditions of the problem are linear. In this case, the temperature field at the end of a cycle must be a linear function of the corresponding field at the beginning of the cycle and of any reservoirs with which the system exchanges heat (e.g., the ambient temperature) during the cycle. If the system is discretized by the finite element or finite difference method, both initial and final states are defined in terms of a finite number of nodal temperatures and hence this linear relationship takes the form of a matrix equation. If the corresponding matrix can be determined, the matrix transfer method, well known in structural mechanics [6], can then be used to determine the evolution of the temperature during subsequent cycles and also permits a direct solution for the steady state. 


\begin{tabular}{|c|c|c|c|}
\hline & $T^{\mathrm{SS}}$ & steady state nodal temperatures \\
\hline \multicolumn{2}{|c|}{$\begin{array}{l}\text { Nomenclature } \\
C, M \text { transfer matrices }\end{array}$} & & $\begin{array}{l}\text { estimated thickness of thermal boundary layer } \\
\text { reservoir temperatures }\end{array}$ \\
\hline $\begin{array}{l}h_{1}, h_{2} \\
k\end{array}$ & $\begin{array}{l}\text { heat transfer coefficients } \\
\text { thermal diffusivity }\end{array}$ & $\tau_{\mathrm{TMM}}$ & $\begin{array}{l}\text { computing time using the transfer matrix } \\
\text { method }\end{array}$ \\
\hline$m$ & number of independent thermal reservoirs & $\tau_{\text {simulati }}$ & \\
\hline$n$ & number of nodes in the discretization & & computing time using simulation \\
\hline$t$ & time & $\omega$ & frequency of periodic heating \\
\hline $\begin{array}{l}t_{0} \\
\boldsymbol{T}^{(p)}\end{array}$ & $\begin{array}{l}\text { time period } \\
\text { vector of nodal temperatures at the beginning }\end{array}$ & & \\
\hline
\end{tabular}

\section{The transfer matrix method}

We suppose that the temperature field at the beginning of the $p$ th cycle is defined by the $n$-vector of nodal temperatures $\boldsymbol{T}^{(p)}$, where $n$ is the number of nodes. During one cycle, various boundaries of the system exchange heat with $m$ reservoirs at temperatures $\Theta_{k}$ $(k=1, m)$ during the several parts of the cycle. The nodal temperatures at the end of the cycle $\boldsymbol{T}^{(p+1)}$ must be a linear function of $\boldsymbol{T}^{(p)}$ and the $\Theta_{k}$. This relation can be written in the general linear form

$\boldsymbol{T}^{(p+1)}=\boldsymbol{M} \boldsymbol{T}^{(p)}+\boldsymbol{C} \Theta$,

where $\Theta$ is the vector of reservoir temperatures $\Theta_{k}, \boldsymbol{M}$ is an $n \times n$ matrix and $\boldsymbol{C}$ is an $n \times m$ matrix.

If the matrices $\boldsymbol{M}$ and $\boldsymbol{C}$ could be determined, Eq. (1) could be used sequentially to determine the nodal temperatures $\boldsymbol{T}_{(p)}$ after any number of heating cycles. Also, the steady periodic state $\boldsymbol{T}^{\mathrm{SS}}$ could be determined directly since we would then have

$\boldsymbol{T}^{(p+1)}=\boldsymbol{T}^{(p)}=\boldsymbol{T}^{\mathrm{SS}}$

and hence

$(\boldsymbol{M}-\boldsymbol{I}) \boldsymbol{T}^{\mathrm{SS}}=-\boldsymbol{C} \Theta$

from Eqs. (1) and (2), where $\boldsymbol{I}$ is the identity matrix.

\subsection{Determining the transfer matrices}

The matrices $\boldsymbol{M}$ and $\boldsymbol{C}$ can be determined by making $(n+m)$ linearly independent simulations of a single cycle. For example, if we simulate a single cycle with initial and boundary conditions

$T_{i}^{(0)}=\delta_{i j}, \quad \Theta_{k}=0$,

where $\delta_{i j}$ is the Kronecker delta, the nodal temperatures at the end of the cycle, $T_{i}^{(1)}$ will define the $j$ th row of $\boldsymbol{M}$. Similarly, if we use the conditions

$T_{i}^{(0)}=0, \quad \Theta_{k}=\delta_{k l}$,

$T_{i}^{(1)}$ will define the $l$ th row of $\boldsymbol{C}$.
It is relatively easy to automate the process of making this sequence of runs and extracting the corresponding matrix elements. The computational effort involved is equivalent to that of performing a direct simulation for $(n+m)$ cycles and hence it will be cost effective if the process under consideration involves a larger number of cycles. However, notice that once the transfer matrices have been determined, they permit the response of the system to be obtained for a range of initial and boundary conditions, whereas a direct simulation would require that the program be restarted from zero if any of these conditions were to change.

\section{Example}

To illustrate the use of the method, we consider the simple two-dimensional example of Fig. 1, which also shows the finite element mesh used. The edge $\mathrm{CD}$ is assumed to make thermal contact with a reservoir whose temperature alternates between 300 and $1300 \mathrm{~K}$ with a period $t_{0}=3.5 \mathrm{~ms}$, as shown in Fig. 2 . The heat transfer coefficient at this boundary is assumed to be $h_{1}=4000$

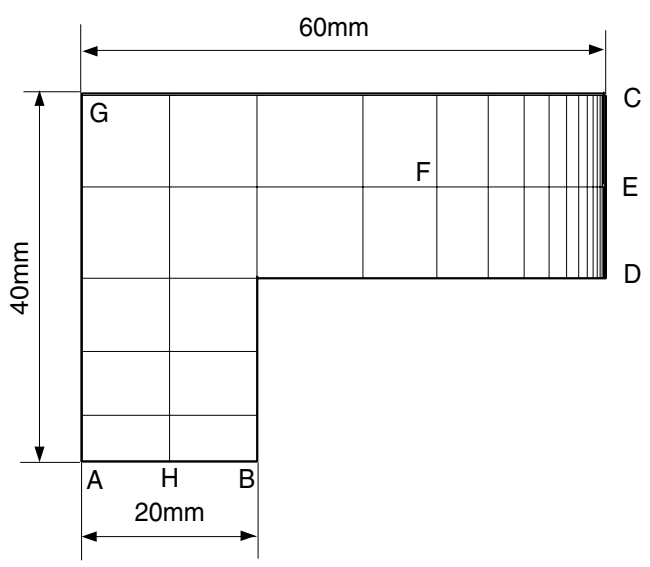

Fig. 1. Geometry of the example problem. 


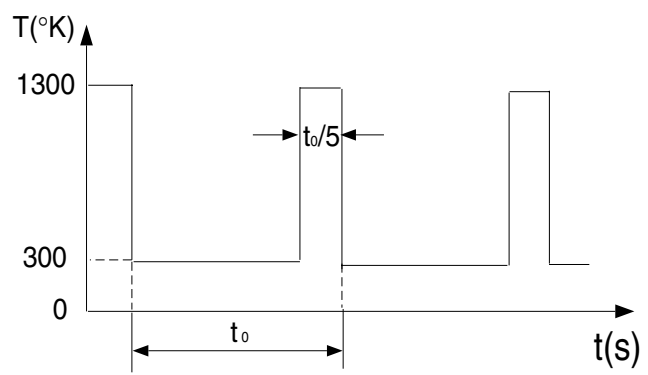

Fig. 2. Time history of the temperature of the reservoir contacting the boundary $C D$.

$\mathrm{W} / \mathrm{m}^{2} \mathrm{~K}$. The edge $A B$ exchanges heat with a reservoir at $300 \mathrm{~K}$ through a heat transfer coefficient $h_{2}=$ $25 \mathrm{~W} / \mathrm{m}^{2} \mathrm{~K}$. The remaining boundaries are insulated and the initial temperature is assumed to be uniform and equal to $300 \mathrm{~K}$.

The periodic boundary condition causes significant variation in temperature during each cycle in a thin thermal boundary layer near to the edge $C D$, necessitating the use of an appropriately refined mesh in this region. The boundary layer is so thin that the temperature field is almost one-dimensional in this region and hence the layer thickness can be estimated from the simpler problem of a half plane $x>0$ whose surface temperature varies sinusoidally as $T(0, t)=T_{0} \cos (\omega t)$. In this case, the steady periodic solution is [7]

$T(x, t)=T_{0} \exp \left(-x \sqrt{\frac{\omega}{2 k}}\right) \cos \left(\omega t-x \sqrt{\frac{\omega}{2 k}}\right)$,

where $k$ is the thermal diffusivity. The temperature oscillation has decayed to less than $1 \%$ of $T_{0}$ within a distance

$\chi=2 \pi \sqrt{\frac{2 k}{\omega}}=2 \sqrt{\pi k t_{0}}$,

where we note that $\omega=2 \pi / t_{0}$. For the present problem, using the material properties of steel $\left(k=10.9 \mathrm{~mm}^{2} / \mathrm{s}\right)$, this gives a value $\chi \approx 0.7 \mathrm{~mm}$. A relatively coarse mesh is sufficient outside this region. We used a biased mesh adjacent to the edge $C D$ giving seven elements in the range $0<x<\chi$ and a smallest element (immediately adjacent to $C D)$ of width $0.026 \mathrm{~mm}(0.037 \chi)$.

Fig. 3 shows the temperature at several nodes near to the heated surface during the first few cycles. These results were obtained by direct numerical simulation, using the finite element package ABAQUS and the mesh of Fig. 1. The temperature shows a typical oscillatory behavior superposed on a gradual increasing temperature which penetrates to greater depths as time progresses.

The same finite element program was used to determine the transfer matrices, using the strategy described

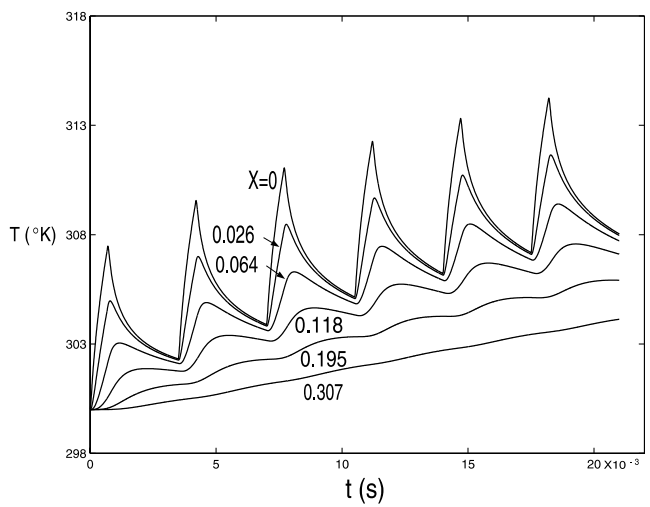

Fig. 3. Time dependence of the temperature at several on the line $E F$ during the first few cycles. The distance from the heated surface is denoted by $x$.

in Section 2.1. These matrices were then used to determine the evolution of the temperature at selected nodes at the same point in successive cycles. The results for the first 5000 cycles (solid lines) are compared with those of the direct simulation program (circles) in Fig. 4 for the points identified as $E, F, H$ in Fig. 1. The agreement is extremely good as we should expect, since mathematically the methods are formally equivalent. The only possible source of computational difference lies in roundoff errors in the computations.

It was not practical to run the simulation program much beyond 5000 cycles, since each cycle takes approximately $2 \mathrm{~min}$ on a workstation. However, once the transfer matrices have been established, the calculation of additional cycles is extremely rapid. Fig. 5 shows results for the first 60,000 heating cycles obtained in this way and show that the results tend asymptotically to a steady periodic state.

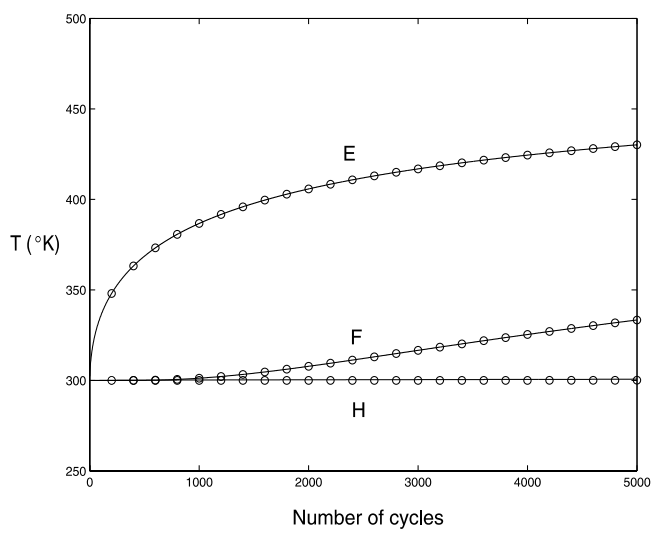

Fig. 4. Comparison of the results of the transfer matrix method (solid line) and numerical simulation (circles) during the first 5000 cycles at the points $E, F, H$ in Fig. 1. 


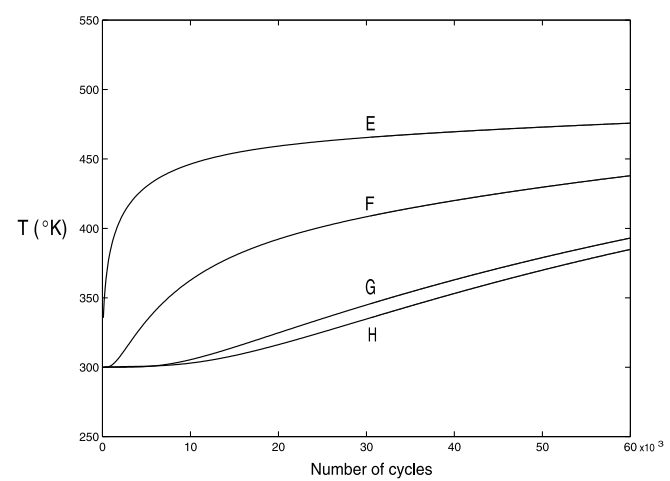

Fig. 5. Long time evolution of the temperatures at the points $E, F, G, H$ in Fig. 1 .

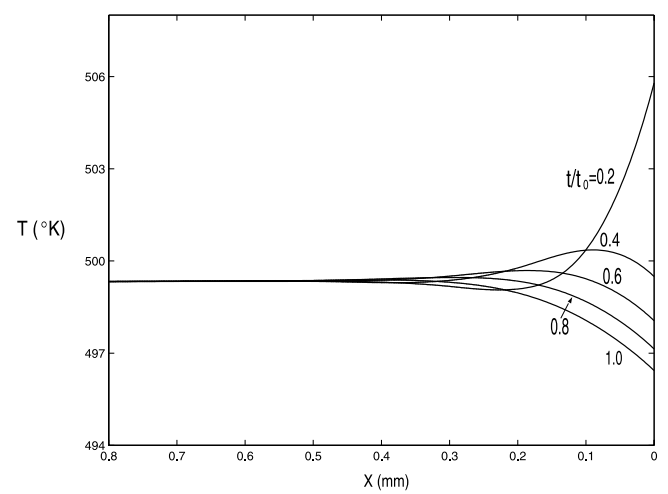

Fig. 6. Temperature variation along the line $E F$ at various points during the steady state cycle.

The steady periodic solution was obtained directly from the transfer matrices using Eq. (3). Input of these nodal temperatures into one cycle of the simulation program then provides the complete steady periodic solution. Fig. 6 shows the variation along the line $E F$ at various points during the steady state cycle.

\subsection{Numerical efficiency of the method}

As explained in Section 2.1, the establishment of the transfer matrices requires that the simulation program be run for $(m+n)$ independent cycles of heating, where $n$ is the number of nodes in the discretization and $m$ is the number of independent reservoirs. Once the transfer matrices are known, the matrix operations defined by Eqs. (1) or (2) require very little computational effort. It follows that the computing time required for the transfer matrix solution $\tau_{\mathrm{TMM}}$ and the simulation $\tau_{\text {simulation }}$ will be approximately in the ratio

$\frac{\tau_{\mathrm{TMM}}}{\tau_{\text {simulation }}} \approx \frac{m+n}{p}$,

where $p$ is the number of cycles of heating to be modeled. For the present example, we used 72 nodes and 2 reservoirs, so the transfer matrix method was over 800 times more efficient than the simulation for the calculation of 60,000 cycles of heating.

\section{Conclusions}

The transfer matrix method discussed in this paper provides a very efficient method for the analysis of linear periodic heat transfer problems. It is particularly suitable for cases where the transient response is required over a large number of cycles or where the same system is to be analyzed for several sets of initial or boundary temperatures.

\section{Acknowledgements}

The authors are pleased to acknowledge support from the Nippon Steel Company and from the National Science Foundation under contract number CMS9619527.

\section{References}

[1] J.R. Howard, A.E. Sutton, An analogue study of heat transfer through periodic contacting surfaces, Int. J. Heat Mass Transfer 13 (1970) 173-183.

[2] W.Y.D. Yuen, Heat conduction in sliding solids, Int. J. Heat Mass Transfer 31 (1988) 637-645.

[3] S.K. Das, R.K. Sahoo, Second law analysis of a cyclic regenerator in presence of longitudinal heat conduction in matrix, Heat Mass Transfer 34 (1999) 395-403.

[4] E. Hahne, M. Hornberger, Experience with a solar heating ATES system for a university building, ASME J. Solar Energy Eng. 116 (1994) 88-93.

[5] B.A.K. Abu-Hijleh, Numerical solution of periodic heat transfer in an anisotropic cylinder subject to asymmetric heat flux, Heat Transfer Eng. 19 (1998) 68-74.

[6] A. Tesar, L. Fillo, Transfer Matrix Method, Kluwer Academic Publishers, Dordrecht, Netherlands, 1988.

[7] H. Carslaw, J.C. Jaeger, The Conduction of Heat in Solids, second ed., Clarendon Press, Oxford, 1959, pp. 64-70. 